T. Ichinose

Nagoya Math. J.

Vol. 50 (1973), 185-198

\title{
OPERATORS ON TENSOR PRODUCTS OF $\mathscr{L}_{1}, \mathscr{L}_{2}$ AND $\mathscr{L}_{\infty}$ SPACES
}

Dedicated to Professor Yoneichiro Sakaki on his 60th birthday, March 5, 1973

\author{
TAKASHI ICHINOSE*
}

\section{Introduction}

Let $A$ and $B$ be densely defined closed linear operators in complex Banach spaces $X, Y$, respectively, with nonempty resolvent sets. Then for a class of polynomials $P(\xi, \eta)$ the spectral mapping theorem has been established by the author [9] (cf. [7], [8]):

$$
P(\sigma(A), \sigma(B))=\sigma(P(A \otimes I, I \otimes B))=\sigma(\tilde{P}(A \otimes I, I \otimes B)),
$$

where $\tilde{P}(A \otimes I, I \otimes B)$ is a maximal extension of $P(A \otimes I, I \otimes B)$ in $X \hat{\otimes}_{\alpha} Y$, the completion of $X \otimes Y$ with respect to a uniform reasonable norm $\alpha$. Another investigation has recently been made by $M$. Reed and $B$. Simon [17].

The aim of this work is to extend the spectral mapping theorem to a much larger class of polynomials $P(\xi, \eta)$, when both $X$ and $Y$ are $\mathscr{L}_{1}$, $\mathscr{L}_{2}$ or $\mathscr{L}_{\infty}$ spaces of J. Lindenstrauss and A. Pełczyński [13] and one of $A$ and $B$ is a scalar type spectral operator (see [2]). In contrast to the results in [9], the set $P(\sigma(A), \sigma(B)$ ) may not always be closed (cf. [17]).

The theory applies to the operators of the form $A \otimes I+I \otimes B$, which include not only the elliptic and parabolic differential operators but also the hyperbolic differential operators. A new meaning is given thereby to the method of separation of variables for partial differential equations (cf. [3]).

Section 1 is concerned with some definitions and results on $\mathscr{L}_{p}$ spaces and tensor products. Our main results are formulated in Section 2. Section 3 is devoted, in particular, to the operators of the forms $A \otimes I+I \otimes B$ and $A \hat{\otimes}_{\alpha} I+I \hat{\otimes}_{\alpha} B$. In Section 4, we refer to some applications of the results.

Received August 4, 1972.

* The author would like to express his hearty thanks to Professor T. Matsuzawa for several valuable discussions. 
For the basic facts on topological linear spaces and tensor products, see [11], [4], [5] and [18].

\section{Preliminary Results}

We shall start by summarizing some useful results on $\mathscr{L}_{p}$ spaces and tensor products.

\section{1. $\mathscr{L}_{p}$ spaces}

J. Lindenstrauss and A. Pelczyński [13] have introduced the $\mathscr{L}_{p}$ spaces. We shall only concern with the cases $p=1,2$ and $\infty$ in this work.

Two Banach spaces $E$ and $F$ are isomorphic if there exists an invertible bounded linear operator of $E$ onto $F$. The distance $d(E, F)$ of two isomorphic Banach spaces is defined by inf $\left(\|T\|\left\|T^{-1}\right\|\right)$, where the infimum is taken over all invertible bounded linear operators $T$ of $E$ onto $F$.

By $L_{p}(\mu)=L_{p}(\Omega, \mathscr{B}, \mu), 1 \leq p \leq \infty$, we denote the Banach space of all equivalence classes of measurable functions on some measure space $(\Omega, \mathscr{B}, \mu)$ whose $p$-th power is integrable (resp. essentially bounded if $p=\infty)$. If $(\Gamma, \mathscr{B}, \mu)$ is the discrete measure space on a set $\Gamma$ with $\mu(\{\gamma\})=1$ for every $\gamma \in \Gamma$, we denote $L_{p}(\mu)$ by $\ell_{p}(\Gamma)$. If $\Gamma=\{1,2, \cdots, n\}$, we denote $\ell_{p}(\Gamma)$ by $\ell_{p}^{n}$. The subspace of $\ell_{\infty}(\Gamma)$ of those $f \in \ell_{\infty}(\Gamma)$ for which the set $\{\gamma \in \Gamma ;|f(\gamma)| \geq \varepsilon\}$ is finite for every $\varepsilon>0$ is denoted by $c_{0}(\Gamma)$. For a compact Hausdorff space $K$, we denote by $C(K)$ the Banach space of all continuous functions on $K$.

A Banach space $E$ is said to be an $\mathscr{L}_{p, \lambda}$ space, $1 \leq p \leq \infty, 1 \leq \lambda<\infty$, if for each finite-dimensional subspace $F \subset E$ there exists a finite-dimensional subspace $G$ with $F \subset G \subset E$ such that $d\left(G, \ell_{p}^{n}\right) \leq \lambda$, where $n=\operatorname{dim} G$, the dimension of $G$,

A Banach space $E$ is said to be an $\mathscr{L}_{p}$ space, $1 \leq p \leq \infty$, if it is an $\mathscr{L}_{p, \lambda}$ space for some $\lambda \geq 1$.

It is known [13] that the Banach spaces $L_{p}(\mu)$ (resp. $C(K)$ ) are $\mathscr{L}_{p, \lambda}$ (resp. $\mathscr{L}_{\infty, \lambda}$ ) spaces for every $\lambda>1$, but for $1 \leq p \leq \infty, p \neq 2$, there exist $\mathscr{L}_{p}$ spaces which are not isomorphic to the spaces $L_{p}(\mu)$. For $p=2$, the class of $\mathscr{L}_{2}$ spaces coincides with the class of spaces isomorphic to Hilbert spaces, so that an $\mathscr{L}_{2}$ space can be considered to be endowed with an inner product.

A closed subspace $F$ of a Banach space $E$ is said to be a complemented subspace if there is a continuous projection of $E$ onto $F$. 
A complemented subspace of an $\mathscr{L}_{p}$ space $(1 \leq p \leq \infty)$ which is not isomorphic to a Hilbert space is an $\mathscr{L}_{p}$ space [14]. Every $\mathscr{L}_{p}$ space $(1 \leq p \leq \infty)$ is isomorphic to a subspace of a space $L_{p}(\mu)$ for some measure $\mu[13]$. Every Banach space $E$ is an $\mathscr{L}_{p}$ space $(1 \leq p \leq \infty)$ if and only if its dual $X^{\prime}$ is an $\mathscr{L}_{p}$, space with $1 / p+1 / p^{\prime}=1$ [14].

By a bounded Boolean algebra $\mathscr{E}$ of projections in a Banach space $Z$, we mean a Boolean algebra of commuting continuous projection $E$ in $Z$ such that $\sup \|E\|<K$ for all $E \in \mathscr{E}$ (see [2]).

We shall make use of a result of J. Lindenstrauss and A. Pelczyński on unconditional Schauder decompositions of $\mathscr{L}_{1}$ and $\mathscr{L}_{\infty}$ spaces [13, Corollary 8 to Theorem 6.1], stated in the following form we need.

THEOREM 1.1. Let $Z$ be an $\mathscr{L}_{1}$ (resp. $\mathscr{L}_{\infty}$ ) space and let $\mathscr{E}$ be a bounded Boolean algebra of projections. Then there exists a positive constant $M_{1}$ (resp. $M_{\infty}$ ) such that for every finite family $\left\{E_{k}\right\}_{k=1}^{s}$ of disjoint projections in $\mathscr{E}$

$$
\begin{gathered}
\sum_{k=1}^{s}\left\|E_{k} z\right\| \leq M_{1}\left\|\sum_{k=1}^{s} E_{k} z\right\|, \quad z \in Z \\
\text { (resp. } \left.\left\|\sum_{k=1}^{s} E_{k} z\right\| \leq M_{\infty} \max _{1 \leq k \leq s}\left\|E_{k} z\right\|, z \in Z\right) .
\end{gathered}
$$

\subsection{Tensor Products}

Let $X$ and $Y$ be complex Banach spaces and $X^{\prime}, Y^{\prime}$ their dual spaces. Let $X \otimes Y$ be the algebraic tensor product of $X$ and $Y$ and $X \hat{\otimes}_{\alpha} Y$ its completion with respect to a uniform reasonable norm $\alpha$ on $X \otimes Y$.

Suppose that $A: \mathrm{D}[A] \subset X \rightarrow X$ and $B: D[B] \subset Y \rightarrow Y$ are densely defined closed linear operators with nonempty resolvent sets $\rho(A), \rho(B)$ and with spectra $\sigma(A), \sigma(B)$. Assume further that $B$ is a scalar type spectral operator with the countably additive resolution $E$ of the identity [2]. We may assume $\sigma(B)$ nonempty. The identity operators in both $X$ and $Y$ will be denoted by the same $I$.

To each polynomial of degrees $m$ in $\xi$ and $n$ in $\eta$

$$
P(\xi, \eta)=\sum c_{j k} \xi^{j} \cdot \eta^{k},
$$

we assign two kinds of polynomial operators defined densely in $X \hat{\otimes}_{\alpha} Y$

$$
P(A \otimes I, I \otimes B)=\sum c_{j k} A^{j} \otimes B^{k}
$$

with domain $D\left[A^{m}\right] \otimes D\left[B^{n}\right]$ and

$$
\sum c_{j k} A^{j} \hat{\otimes}_{\alpha} B^{k}
$$

with domain $\bigcap_{j, k ; c_{j k} \neq 0} D\left[A^{j} \hat{\otimes}_{\alpha} B^{k}\right]$, where $A^{j} \hat{\otimes}_{\alpha} B^{k}$ denotes a maximal 
extension of $A^{j} \otimes B^{k}$ in $X \hat{\otimes}_{\alpha} Y$. Maximal extensions of the operators (1.2) and (1.3) are denoted by $\tilde{P}(A \otimes I, I \otimes B),\left(\sum c_{j k} A^{j} \hat{\otimes}_{\alpha} B^{k}\right)^{\sim}$, respectively (see [12], [7], [8] and [9]).

In order to establish our main results, we shall restrict the Banach spaces $X \hat{\otimes}_{\alpha} Y$ concerned to the following three cases:

$\left(\alpha_{1}\right)$ both $X$ and $Y$ are $\mathscr{L}_{1}$ spaces and $\alpha$ is the greatest reasonable norm $\pi$;

$\left(\alpha_{2}\right)$ both $X$ and $Y$ are $\mathscr{L}_{2}$ spaces and $\alpha$ is the uniform crossnorm $\alpha_{0}$ for which $X \hat{\otimes}_{\alpha} Y$ is an $\mathscr{L}_{2}$ space;

$\left(\alpha_{\infty}\right)$ both $X$ and $Y$ are $\mathscr{L}_{\infty}$ spaces and $\alpha$ is the smallest reasonable norm $\varepsilon$

In cases $\left(\alpha_{2}\right)$ and $\left(\alpha_{\infty}\right)$, the norms $\alpha_{0}$ and $\varepsilon$ are faithful. For case $\left(\alpha_{1}\right)$, however, the author is unaware whether or not the norm $\pi$ there is faithful. This is certainly true if $X$ or $Y$ satisfies the condition of approximation [4]. It is known [10] that a separable $\mathscr{L}_{p}$ space $(1 \leq p \leq \infty)$ has a Schauder basis. Therefore we can assert in case $\left(\alpha_{1}\right)$ that if $X$ and $Y$ are besides separable, then the norm $\pi$ there is faithful (e.g. [7]).

Thus, as is the case in all applications, we assume for simplicity further that the norm $\pi$ is faithful in case $\left(\alpha_{1}\right)$ whenever both $X$ and $Y$ are $\mathscr{L}_{1}$ spaces.

For faithful $\alpha, A^{j} \hat{\otimes}_{\alpha} B^{k}$ is nothing but the closure of $A^{j} \otimes B^{k}$ in $X \hat{\otimes}_{\alpha} Y$, and the same is true for $\tilde{P}(A \otimes I, I \otimes B)$ and $\left(\sum c_{j k} A^{j} \hat{\otimes}_{\alpha} B^{k}\right)^{-}$.

We remark (cf. [14], [6]) that under the condition $\left(\alpha_{1}\right)$ (resp. $\left(\alpha_{2}\right)$, $\left.\left(\alpha_{\infty}\right)\right), X \hat{\otimes}_{\alpha} Y$ is also an $\mathscr{L}_{1}$ (resp. $\mathscr{L}_{2}, \mathscr{L}_{\infty}$ ) space.

To prove Proposition 2.1, we shall need

LEMMA 1.2. Suppose the space $X \hat{\otimes}_{\alpha} Y$ satisfies the condition $\left(\alpha_{1}\right)$, $\left(\alpha_{2}\right)$ or $\left(\alpha_{\infty}\right)$. If $Y_{1}$ is a complemented subspace of $Y$, then $X \hat{\otimes}_{\alpha} Y_{1}$ is a complemented subspace of $X \hat{\otimes}_{\alpha} Y$. Therefore, if $Y=\sum_{k=1}^{s} \oplus Y_{k}$ is a topological direct sum, then $X \hat{\otimes}_{\alpha} Y=\sum_{k=1}^{s} \oplus\left(X \hat{\otimes}_{\alpha} Y_{k}\right)$.

Proof. It is trivial for case $\left(\alpha_{2}\right)$. For cases $\left(\alpha_{1}\right)$ and $\left(\alpha_{\infty}\right)$, note that the norms $\pi$ and $\varepsilon$ are $\otimes$-norms in the sense of A. Grothendieck [5], so that they are defined for every pair of Banach spaces $X$ and $Y$. Let $P$ be the continuous projection of $Y$ onto $Y_{1}$, and $J$ the injection of $Y_{1}$ into $Y$. Then, since $\alpha$ is a $\otimes$-norm, $I \hat{\otimes}_{\alpha} P$ (resp. $I \hat{\otimes}_{\alpha} J$ ) is a continuous linear operator of $X \hat{\otimes}_{\alpha} Y$ (resp. $X \hat{\otimes}_{\alpha} Y_{1}$ ) into $X \hat{\otimes}_{\alpha} Y_{1}$ (resp. $X \hat{\otimes}_{\alpha} Y$ ). We have $I \hat{\otimes}_{\alpha} P u=(I \otimes P) u=u$ on $X \otimes Y_{1}$. Since $(I \otimes P)(I \otimes J) u=u$ on 
$X \otimes Y_{1}$, it follows by continuity that $\left(I \hat{\otimes}_{\alpha} P\right)\left(I \hat{\otimes}_{\alpha} J\right) u=u$ on $X \hat{\otimes}_{\alpha} Y_{1}$, which implies that the range of $I \hat{\otimes}_{\alpha} P$ is $X \hat{\otimes}_{\alpha} Y_{1}$. Clearly $\left(I \hat{\otimes}_{\alpha} P\right)^{2}=$ $I \hat{\otimes}_{\alpha} P$. Thus $I \hat{\otimes}_{\alpha} P$ is a continuous projection of $X \hat{\otimes}_{\alpha} Y$ onto $X \hat{\otimes}_{\alpha} Y_{1}$, so that $X \hat{\otimes}_{\alpha} Y_{1}$ is a complemented subspace of $X \hat{\otimes}_{\alpha} Y$. Q.E.D.

\section{Spectral Mapping Theorem}

For the spaces $X \hat{\otimes}_{\alpha} Y$ and the operators $A$ and $B$ described in Section 1 , we shall formulate the spectral mapping theorem for the polynomial operators (1.2) and (1.3).

Throughout, the following convention will be used. Given two subsets $F_{1}$ and $F_{2} \neq \emptyset$ of the complex plane $C$ and a polynomial $P(\xi, \eta)$ of degrees $m \geq 1$ in $\xi$ and $n$ in $\eta$, we can define $P\left(F_{1}, F_{2}\right)$ and its closure $\overline{P\left(F_{1}, F_{2}\right)}$ in an obvious way if $F_{1}$ is not empty, and otherwise we set $P\left(F_{1}, F_{2}\right)=\overline{P\left(F_{1}, F_{2}\right)}=\emptyset$.

Proposition 2.1. Let $\alpha$ be a faithful uniform reasonable norm on $X \otimes Y . \quad$ Suppose that the space $X \hat{\otimes}_{\alpha} Y$ satisfies the condition $\left(\alpha_{1}\right),\left(\alpha_{2}\right)$ or $\left(\alpha_{\infty}\right)$, and that $A$ and $B$ are those operators described in Section 1 . Let $P(\xi, \eta)$ be a polynomial of degrees $m \geq 1$ in $\xi$ and $n$ in $\eta$ such that if $\sigma(A)$ is nonempty, $\overline{P(\sigma(A), \sigma(B))}$ does not cover the whole complex plane C. Then for $\lambda \notin \overline{P(\sigma(A), \sigma(B))}$, we have

$$
\lambda \notin \sigma(P(A \otimes I, I \otimes B))=\sigma(\tilde{P}(A \otimes I, I \otimes B)),
$$

provided that $P(A, \eta)-\lambda I$, with $\eta \in \sigma(B)$, has an everywhere defined continuous inverse $(P(A, \eta)-\lambda I)^{-1}$ which is uniformly bounded on $\sigma(B)$.

Proof. First note that the complement of $\sigma(B)$ has $E$-measure zero so that $E(\sigma(B))=I$. We shall show for $\lambda \notin \overline{P(\sigma(A), \sigma(B))}$ that $\tilde{P}(A \otimes I, I \otimes B)-\lambda I \hat{\otimes}_{\alpha} I$ has an everywhere defined continuous inverse. To do so, we must establish that the improper Riemann integral

$$
\int_{\sigma(B)}\left[(P(A, \eta)-\lambda I)^{-1} \otimes E(d \eta)\right] v, \quad v \in X \otimes Y,
$$

exists a an element of $X \hat{\otimes}_{\alpha} Y$ and defines a continuous linear operator of $X \otimes Y \subset X \hat{\otimes}_{\alpha} Y$ into $X \hat{\otimes}_{\alpha} Y$, taking the condition for the spaces $X \hat{\otimes}_{\alpha} Y$ into consideration.

Let $\sigma$ be an arbitrary Borel set and $e=\sigma \cap \sigma(\mathrm{B})$. Let $\left\{e_{1}, \cdots, e_{s}\right\}$ be an arbitrary finite decomposition of $\sigma$ into disjoint Borel sets. We may 
assume $e_{k} \cap e \neq \emptyset$ for all $k, 1 \leq k \leq s$. Set $e_{k}^{\prime}=e_{k} \cap e$ and $\eta_{k} \in e_{k}^{\prime}$ for $k=1,2, \cdots, s$. By assumption, $\left(P\left(A, \eta_{k}\right)-\lambda I\right)^{-1}$ is then uniformly bounded for all $k$ and for all the decompositions $\left\{e_{k}\right\}_{k=1}^{s}$ of $\sigma$ into disjoint Borel sets. Clearly $E(e)=\sum_{k=1}^{s} E\left(e_{k}^{\prime}\right)$. It follows in virtue of Lemma 1.2 that the $I \hat{\otimes}_{\alpha} E\left(e_{k}^{\prime}\right)$ are mutually disjoint continuous projections in $X \hat{\otimes}_{\alpha} Y$ and of $\sigma$ into $I \hat{\otimes}_{\alpha} E(e)=\sum_{k=1}^{s} I \hat{\otimes}_{\alpha} E\left(e_{k}^{\prime}\right)$.

In case $\left(\alpha_{1}\right)$ where $X \hat{\otimes}_{\alpha} Y$ in an $\mathscr{L}_{1}$ space, we obtain by Theorem 1.1 for $v \in X \otimes Y$

$$
\begin{aligned}
\| \sum_{k=1}^{s} & {\left[\left(P\left(A, \eta_{k}\right)-\lambda I\right)^{-1} \otimes E\left(e_{k}^{\prime}\right)\right] v \|_{\pi} } \\
& =\left\|\sum_{k=1}^{s}\left[\left(P\left(A, \eta_{k}\right)-\lambda I\right)^{-1} \otimes I\right]\left[I \otimes E\left(e_{k}^{\prime}\right)\right] v\right\|_{\pi} \\
& \leq \sum_{k=1}^{s}\left\|\left(P\left(A, \eta_{k}\right)-\lambda I\right)^{-1}\right\|\left\|\left[I \otimes E\left(e_{k}^{\prime}\right)\right] v\right\|_{\pi} \\
& \leq C_{1} \sum_{k=1}^{s}\left\|\left[I \otimes E\left(e_{k}^{\prime}\right)\right] v\right\|_{\pi} \\
& \leq C_{1} M_{1}\left\|\sum_{k=1}^{s}\left[I \otimes E\left(e_{k}^{\prime}\right)\right] v\right\|_{\pi} \\
& =C\|[I \otimes E(e)] v\|_{\pi} .
\end{aligned}
$$

In case $\left(\alpha_{2}\right)$, since $X \hat{\otimes}_{\alpha} Y$ is considered as a Hilbert space, we obtain for $v, w \in X \otimes Y$

$$
\begin{aligned}
\mid\left(\sum_{k=1}^{s}\right. & {\left.\left.\left[\left(P\left(A, \eta_{k}\right)-\lambda I\right)^{-1} \otimes E\left(e_{k}^{\prime}\right)\right] v, w\right)\right] \mid } \\
& =\left|\sum_{k=1}^{s}\left(\left[\left(P\left(A, \eta_{k}\right)-\lambda I\right)^{-1} \otimes I\right]\left[I \otimes E\left(e_{k}^{\prime}\right)\right] v,\left[I \otimes E\left(e_{k}^{\prime}\right)\right] w\right)\right| \\
& \leq \sum_{k=1}^{s}\left\|\left(P\left(A, \eta_{k}\right)-\lambda I\right)^{-1}\right\|\left\|\left[I \otimes E\left(e_{k}^{\prime}\right)\right] v\right\|_{\alpha_{0}} \cdot\left\|\left[I \otimes E\left(e_{k}^{\prime}\right)\right] w\right\|_{\alpha_{0}} \\
& \leq C_{1}\left\{\sum_{k=1}^{s}\left(\left[I \otimes E\left(e_{\mathrm{k}}^{\prime}\right)\right] v, v\right)\right\}^{1 / 2}\left\{\sum_{k=1}^{s}\left(\left[I \otimes E\left(e_{k}^{\prime}\right)\right] w, w\right)\right\}^{1 / 2} \\
& =C\|[I \otimes E(e)] v\|_{\alpha_{0}} \cdot\|w\|_{\alpha_{0}} .
\end{aligned}
$$

In case $\left(\alpha_{\infty}\right)$ where $X \hat{\otimes}_{\varepsilon} Y$ is an $\mathscr{L}_{\infty}$ space, by Theorem 1.1 we obtain for $v \in X \otimes Y$

$$
\begin{aligned}
\| \sum_{k=1}^{s} & {\left[\left(P\left(A, \eta_{k}\right)-\lambda I\right)^{-1} \otimes E\left(e_{k}^{\prime}\right)\right] v \|_{\varepsilon} } \\
& =\left\|\sum_{k=1}^{s}\left[I \otimes E\left(e_{k}^{\prime}\right)\right]\left(\sum_{j=1}^{s}\left[\left(P\left(A, \eta_{j}\right)-\lambda I\right)^{-1} \otimes E\left(e_{j}^{\prime}\right)\right] v\right)\right\|_{\varepsilon} \\
& \leq M_{\infty} \max _{1 \leq k \leq s}\left\|\left[\left(P\left(A, \eta_{k}\right)-\lambda I\right)^{-1} \otimes E\left(e_{k}^{\prime}\right)\right] v\right\|_{\varepsilon} \\
& \leq M_{\infty} \max _{1 \leq k \leq s}\left\|\left(P\left(A, \eta_{k}\right)-\lambda I\right)^{-1}\right\|\left\|\left[I \otimes E\left(e_{k}^{\prime}\right)\right] v\right\|_{s} \\
& \leq C_{1} \max _{1 \leq k \leq s}\left\|\left[I \otimes E\left(e_{k}^{\prime}\right)\right] v\right\|_{\varepsilon} \\
& \leq C\|[I \otimes E(e)] v\|_{s} .
\end{aligned}
$$

Here we have used the same symbol $C$ to express different constants independent of the decompositions.

This assures that the integral (2.1) defines a continuous linear operator 
of $X \otimes Y \subset X \hat{\otimes}_{\alpha} Y$ into $X \hat{\otimes}_{\alpha} Y$. We denote its continuous extension to $X \hat{\otimes}_{\alpha} Y$ by $\widetilde{P}_{\lambda}^{-1}(A \otimes I, I \otimes B)$ (with $P_{\lambda}=P-\lambda$ ).

Recalling the definition of the integral with respect to the measure $E$, we can show easily that for $u \in D\left[A^{m}\right] \otimes D\left[B^{n}\right]$

$$
\begin{aligned}
\widetilde{P}_{\lambda}^{-1}(A & \otimes I, I \otimes B)\left[\tilde{P}(A \otimes I, I \otimes B)-\lambda I \hat{\otimes}_{\alpha} I\right] u \\
& =\int_{\sigma(B)}\left[(P(A, \eta)-\lambda I)^{-1} \otimes I\right][P(A \otimes I, I \otimes B)-\lambda I \otimes I][I \otimes E(d \eta)] u \\
& =\int_{\sigma(B)}\left[(P(A, \eta)-\lambda I)^{-1} \otimes I\right][(P(A, \eta)-\lambda I) \otimes I][I \otimes E(d \eta)] u \\
& =\int_{\sigma(B)}[I \otimes E(d \eta)] u \\
& =u .
\end{aligned}
$$

It follows by the continuity of $\widetilde{P}_{\lambda}^{-1}(A \otimes I, I \otimes B)$ that

$$
\widetilde{P}_{\lambda}^{-1}(A \otimes I, I \otimes B)\left[\tilde{P}(A \otimes I, I \otimes B)-\lambda I \hat{\otimes}_{\alpha} I\right] u=u
$$

for all $u$ in the domain of $\tilde{P}(A \otimes I, I \otimes B)$.

Just in the same way, using the closedness of $\tilde{P}(A \otimes I, I \otimes B)$, we can show that

$$
\left[\tilde{P}(A \otimes I, I \otimes B)-\lambda I \hat{\otimes}_{\alpha} I\right] \widetilde{P}_{\lambda}^{-1}(A \otimes I, I \otimes B) v=v
$$

for all $v \in X \hat{\otimes}_{\alpha} Y$.

Thus, $\widetilde{P}_{\lambda}^{-1}(A \otimes I, I \otimes B)$ is the everywhere defined continuous inverse of $\tilde{P}(A \otimes I, I \otimes B)-\lambda I \hat{\otimes}_{\alpha} I$. Q.E.D.

In order to state the spectral mapping theorem for the operator (1.2) and its closure, we introduce a class of polynomials, larger than the one in [9], which will turn out to satisfy the assumptions of Proposition 2.1.

Let $\mathscr{P}^{\prime}(A, B)$ be the class of polynomials $P(\xi, \eta)$ of degrees $m \geq 1$ in $\xi$ and $n$ in $\eta$ satisfying the following condition: for any open neighbourhood $W$ in $C$ of the closure of $P(\sigma(A), \sigma(B)$ ) (when $\sigma(A)$ is empty, take $W=\complement K(0 ; R)$ for any $R>0$, where $K(0 ; R)$ is the closed disc $\{\zeta ;|\zeta| \leq R\}$, there exists a nonempty open set $U$ whose complement $\{U$ is contained in $\rho(A)$ (resp. $\rho(B)$ ) such that

(i) $\quad P(U, \sigma(B)) \subset W$, and

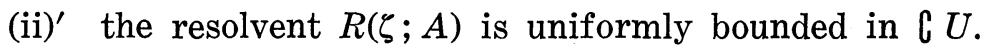

We note that the set $P(\sigma(A), \sigma(B)$ ) is not necessarily closed in $C$ (cf. [9] and [17]). 
Then we have

THEOREM 2.2. Let $\alpha$ be a faithful uniform reasonable norm on $X \otimes Y . \quad$ Suppose the space $X \hat{\otimes}_{\alpha} Y$ satisfies the condition $\left(\alpha_{1}\right),\left(\alpha_{2}\right)$ or $\left(\alpha_{\infty}\right)$. Let $A: D[A] \subset X \rightarrow X$ be a densely defined closed linear operator with $\rho(A) \neq \emptyset$ and let $B: D[B] \subset Y \rightarrow Y$ be a densely defined, closed, scalar type spectral operator with $\rho(B) \neq \emptyset$ and $\sigma(B) \neq \emptyset$. Then for $P \in \mathscr{P}^{\prime}(A, B)$ it holds

$$
\overline{P(\sigma(A), \sigma(B))}=\sigma(P(A \otimes I, I \otimes B))=\sigma(\tilde{P}(A \otimes I, I \otimes B)) .
$$

This means that (2.2) holds valid if $\sigma(A)$ is not empty, and that the spectra of $P(A \otimes I, I \otimes B)$ and its closure $\tilde{P}(A \otimes I, I \otimes B)$ are empty if and only if $\sigma(A)$ is empty.

Proof. Let $P \in \mathscr{P}^{\prime}(A, B)$ be of the form

$$
P(\xi, \eta)=c_{m}(\eta) \xi^{m}+c_{m-1}(\eta) \xi^{m-1}+\cdots+c_{0}(\eta),
$$

where $c_{m}(\eta) \not \equiv 0$. When $\sigma(A)$ and $\sigma(B)$ are nonempty, the inclusion

$$
\overline{P(\sigma(A), \sigma(B))} \subset \sigma(P(A \otimes I, I \otimes B))=\sigma(\tilde{P}(A \otimes I, I \otimes B))
$$

is already shown ([8], [9]).

The proof of the rest of Theorem 2.2 will be reduced to Proposition 2.1. Since the resolvent set $\rho(A)$ is not empty, for $\eta$ fixed

$$
P(A, \eta)=\sum_{j=0}^{m} c_{j}(\eta) A^{j}
$$

is a densely defined closed linear operator in $X$ with domain $D\left[A^{m(\eta)}\right]$, where $m(\eta)$ is the greatest integer, $0 \leq m(\eta) \leq m$, for which $c_{m(\eta)}(\eta) \neq 0$. When $\sigma(A)$ is not empty, we may assume $\overline{P(\sigma(A), \sigma(B))} \neq C$. Then for $\lambda \notin \overline{P(\sigma(A), \sigma(B))}$ (when $\sigma(A)$ is empty, $\lambda$ shall be an arbitrary complex number), we have only to show that $(P(A, \eta)-\lambda I)^{-1}$ is a continuous linear operator defined on the whole $X$ for each $\eta \in \sigma(B)$ and is uniformly bounded on $\sigma(B)$.

Since $P \in \mathscr{P}^{\prime}(A, B)$, there exists by assumption a nonempty open set $U$ such that $|P(\xi, \eta)-\lambda|$ is bounded away from zero on $U \times \sigma(B)$, and such that $R(\xi ; A)$ is uniformly bounded in $\complement U$.

Choose a sufficiently large $R>0$ such that the polynomial $c_{m}(\eta)$ in (2.3) has no zero on $\sigma(B) \cap \complement K(0 ; R-1)$. Then we have $c_{m}=\inf \left|c_{m}(\eta)\right|>0$ for $\eta \in \sigma(B) \cap \complement K(0 ; R)$. Since $P(A, \eta)-\lambda I$ is a closed operator, we have by 
the usual spectral mapping theorem $\sigma(P(A, \eta))=P(\sigma(A), \eta)$ for each $\eta \in \sigma(B)$. It follows that $P(A, \eta)-\lambda I$ has an everywhere defined continuous inverse in $X$ for each $\eta \in \sigma(B)$. It is clear that $(P(A, \eta)-\lambda I)^{-1}$ is uniformly bounded on the compact set $\sigma(B) \cap K(0 ; R)$.

Further for $\eta \in \sigma(B) \cap \complement K(0 ; R)$ we have

$$
P(\xi, \eta)-\lambda=c_{m}(\eta) \prod_{j=1}^{m}\left(\xi-\xi_{j}(\eta)\right),
$$

where none of the $\xi_{j}(\eta)$ lie in $U$. Since $R(\xi ; A)$ is uniformly bounded in $\complement U$, we obtain for $\eta \in \sigma(B) \cap \complement K(0 ; R)$ and for $x \in D\left[A^{m}\right]$

$$
\begin{aligned}
\|(P(A, \eta)-\lambda I) x\| & =\left\|c_{m}(\eta) \prod_{j=1}^{m}\left(A-\xi_{j}(\eta) I\right) x\right\| \\
& \geq c_{m}\left\|\prod_{j=1}^{m}\left(A-\xi_{j}(\eta) I\right) x\right\| \\
& \geq C\|x\|,
\end{aligned}
$$

with a positive constant $C$ independent of $\eta$. Since $D\left[A^{m}\right]$ is the domain of $P(A, \eta)$ for these $\eta,(P(A, \eta)-\lambda I)^{-1}$ is also uniformly bounded on $\sigma(B) \cap \complement K(0 ; R)$. This proves uniform boundedness of $(P(A, \eta)-\lambda I)^{-1}$ on $\sigma(B)$ Q.E.D.

To establish the spectral mapping theorem for the operator (1.3) and its closure, we shall show

THEOREM 2.3. Let $X \hat{\otimes}_{\alpha} Y$ and $A, B$ be as in Theorem 2.2. For $a$ polynomial $P(\xi, \eta)$ of degrees $m \geq 1$ in $\xi$ and $n$ in $\eta$, if there is a complex number $\lambda$ such that the closed operator $P(A, \eta)-\lambda I$ has an everywhere defined continuous inverse for each $\eta \in \sigma(B)$ which is uniformly bounded on $\sigma(B)$, then the closures of the polynomial operators (1.2) and (1.3) coincide.

In particular, for $P \in \mathscr{P}^{\prime}(A, B)$ the above assertion is valid, provided that $\overline{P(\sigma(A), \sigma(B))} \neq C$.

Proof. We must show the closed operator $\left(\sum c_{j k} A^{j} \hat{\otimes}_{\alpha} B^{k}\right)^{\sim}-\lambda I \hat{\otimes}_{\alpha} I$ is one-to-one. Since the norm $\alpha$ considered is always faithful, it suffices to prove that $\tilde{P}\left(A^{\prime} \otimes I^{\prime}, I^{\prime} \otimes B^{\prime}\right)-\lambda I^{\prime} \hat{\otimes}_{\alpha^{\prime}} I^{\prime}$ has a dense range in $X^{\prime} \hat{\otimes}_{\alpha^{\prime}} Y^{\prime}$ (cf. [9]).

Let us note the following facts. The (Banach-space-) adjoint $B^{\prime}$ of $B$ is also a scalar type spectral operator in the dual space $Y^{\prime}$ with the resolution $E^{\prime}$ (=the adjoint of $E$ ) of the identity. The spectrum of a densely defined operator coincides with that of its adjoint, and

$$
P\left(A^{\prime}, \eta\right)-\lambda I^{\prime}=(P(A, \eta)-\lambda I)^{\prime} \quad \text { for } \eta \in \sigma(B)=\sigma\left(B^{\prime}\right) .
$$


In case $\left(\alpha_{1}\right)$ (resp. $\left.\left(\alpha_{2}\right)\right), X^{\prime} \hat{\otimes}_{\alpha^{\prime}} Y^{\prime}$ is an $\mathscr{L}_{\infty}$ (resp. $\left.\mathscr{L}_{2}\right)$ space, because $\pi^{\prime}=\varepsilon\left(\right.$ resp. $\left.\alpha_{0}^{\prime}=\alpha_{0}\right)$. Then we can show just in the same way as in the proof of Proposition 2.1 that $\tilde{P}\left(A^{\prime} \otimes I^{\prime}, I^{\prime} \otimes B^{\prime}\right)-\lambda I^{\prime} \hat{\otimes}_{\alpha^{\prime}} I^{\prime}$ has the range $X^{\prime} \hat{\otimes}_{\alpha^{\prime}} Y^{\prime}$. In case $\left(\alpha_{\infty}\right), X^{\prime} \hat{\otimes}_{\pi} Y^{\prime}$ is an $\mathscr{L}_{1}$ space. Similarly, $\tilde{P}\left(A^{\prime} \otimes I^{\prime}, I^{\prime} \otimes B^{\prime}\right)-\lambda I^{\prime} \hat{\otimes}_{\pi} I^{\prime}$ is seen to have the range $X^{\prime} \hat{\otimes}_{\pi} Y^{\prime}$, so that $P\left(A^{\prime} \otimes I^{\prime}, I^{\prime} \otimes B^{\prime}\right)-\lambda I^{\prime} \otimes I^{\prime}$ maps $D\left[\left(A^{\prime}\right)^{m}\right] \otimes D\left[\left(B^{\prime}\right)^{n}\right]$ onto a dense subspace of $X^{\prime} \otimes Y^{\prime}$ in the norm $\pi$. Since we have $\varepsilon^{\prime} \leq \pi$, it follows that $\tilde{P}\left(A^{\prime} \otimes I^{\prime}, I^{\prime} \otimes B^{\prime}\right)-\lambda I^{\prime} \hat{\otimes}_{\varepsilon^{\prime}} I^{\prime}$ has a dense range in $X^{\prime} \hat{\otimes}_{\varepsilon^{\prime}} Y^{\prime}$. Thus in all the three cases, $\tilde{P}\left(A^{\prime} \otimes I^{\prime}, I^{\prime} \otimes B^{\prime}\right)-\lambda I^{\prime} \hat{\otimes}_{\alpha^{\prime}} I^{\prime}$ is shown to have a dense range in $X^{\prime} \hat{\otimes}_{\alpha^{\prime}} Y^{\prime}$. Q.E.D.

Since the spectrum is unchanged under the closure operation, the following theorem is a direct consequence of Theorems 2.2 and 2.3.

THEOREM 2.4. Under the same assumption as in Theorem 2.2, we have for $P \in \mathscr{P}^{\prime}(A, B)$

$$
\begin{aligned}
\overline{P(\sigma(A), \sigma(B))} & =\overline{P\left(\sigma\left(A \hat{\otimes}_{\alpha} I\right), \sigma\left(I \hat{\otimes}_{\alpha} B\right)\right)} \\
& =\sigma(P(A \otimes I, I \otimes B))=\sigma(\tilde{P}(A \otimes I, I \otimes B)) \\
& =\sigma\left(\sum c_{j k} A^{j} \hat{\otimes}_{\alpha} B^{k}\right)=\sigma\left(\left(\sum c_{j k} A^{j} \hat{\otimes}_{\alpha} B^{k}\right)^{\sim}\right) .
\end{aligned}
$$

\section{Operators $\boldsymbol{A} \otimes \boldsymbol{I}+\boldsymbol{I} \otimes \boldsymbol{B}$ and $\boldsymbol{A} \hat{\otimes}_{\alpha} \boldsymbol{I}+\boldsymbol{I} \hat{\otimes}_{\alpha} \boldsymbol{B}$}

In this section, we consider in particular the operators of the forms $A \otimes I+I \otimes B$ and $A \hat{\otimes}_{\alpha} I+I \hat{\otimes}_{\alpha} B$, which are of especial importance in applications.

As a direct consequence of Theorem 2.4 for $P(\xi, \eta)=\xi+\eta$, the results of Ju. M. Berezanskiř [1] and L. and K. Maurin [15] for selfadjoint operators are generalized as follows (cf. [9]).

THEOREM 3.1. Let $\alpha, X \hat{\otimes}_{\alpha} Y$ and $A, B$ be those described in Section 1. Suppose further that if $\sigma(A)$ is not empty, we have $\|R(\zeta ; A)\| \leq C_{\delta}$ outside $U_{\delta}=\{\zeta$; $\operatorname{dist}(\zeta, \sigma(A))<\delta\}$ for any $\delta>0$ and that if $\sigma(A)$ is empty, for any $R>0$ there exists a nonempty open set $U$ for which $U+\sigma(B) \subset$ $\complement K(0 ; R)$ and $\|R(\zeta ; A)\| \leq C_{R}$ in $\complement U$. Here, $C_{\delta}$ and $C_{R}$ are constants depending only on $\delta, R$, respectively. Then the spectra of $A \otimes I+I \otimes B$, $A \hat{\otimes}_{\alpha} I+I \hat{\otimes}_{\alpha} B$ and their closures are empty if and only if $\sigma(A)$ is empty. If $\sigma(A)$ is not empty, it holds

$$
\begin{aligned}
\overline{\sigma(A)+\sigma(B)} & =\overline{\sigma\left(A \hat{\otimes}_{\alpha} I\right)+\sigma\left(I \hat{\otimes}_{\alpha} B\right)} \\
& =\sigma(A \otimes I+I \otimes B)=\sigma\left((A \otimes I+I \otimes B)^{\sim}\right) \\
& =\sigma\left(A \hat{\otimes}_{\alpha} I+I \hat{\otimes}_{\alpha} B\right)=\sigma\left(\left(A \hat{\otimes}_{\alpha} I+I \hat{\otimes}_{\alpha} B\right)^{\sim}\right) .
\end{aligned}
$$


Proof. For $\sigma(A)$ empty, the assertion of Theorem 3.1 is clear from Theorems 2.2 and 2.4 for $P(\xi, \eta)=\xi+\eta$. If $\sigma(A)$ is not empty, we may assume $\overline{\sigma(A)+\sigma(B)} \neq C$. Let $\lambda \notin \overline{\sigma(A)+\sigma(B)}$, so that $\delta=\operatorname{dist}(\lambda, \sigma(A)+$ $\sigma(B))>0$. Choose $U=U_{\delta / 2} \equiv\{\xi$; dist $(\xi, \sigma(A))<\delta / 2\}$. Then $|\xi+\eta-\lambda|$ is bounded away from zero on $U \times \sigma(B)$, and by assumption $R(\xi ; A)$ is uniformly bounded in $\complement U$. Thus the same argument as in the proof of Theorem 2.2 yields the desired assertion. Q.E.D.

We consider now when the closure of $A \otimes I+I \otimes B$ coincides with $A \hat{\otimes}_{\alpha} I+I \hat{\otimes}_{\alpha} B$. They coincide if and only if $A \hat{\otimes}_{\alpha} I+I \hat{\otimes}_{\alpha} B$ is closed in $X \hat{\otimes}_{\alpha} Y$.

The following theorem is an extension of the part (1) of Theorem 4.6 in [9]. The sector $\{\zeta ;|\arg \zeta| \leq \theta\}$ is denoted by $S(\theta)$.

THEOREM 3.2. Let $\alpha, X \hat{\otimes}_{\alpha} Y$ and $A, B$ be those described in Section 1. Suppose, for some $\theta_{A}$ and $\theta_{B}$ with $0 \leq \theta_{A}, \theta_{B}<\pi$ and $0 \leq \theta_{A}+\theta_{B}<\pi$, that $\rho(A)$ contains the complement of the sector $S\left(\theta_{A}\right)$ and $\|\zeta R(\zeta ; A)\| \leq M_{\theta}$ outside $S(\theta)$ for each $\theta$ with $\theta_{A}<\theta<\pi$, where $M_{\theta}$ is a constant depending only on $\theta$, and that $\rho(B)$ contains the complement of the sector $S\left(\theta_{B}\right)$. Then the closure of $A \otimes I+I \otimes B$ coincides with $A \hat{\otimes}_{\alpha} I+I \hat{\otimes}_{\alpha} B$. The spectra of $A \otimes I+I \otimes B$ and $A \hat{\otimes}_{\alpha} I+I \hat{\otimes}_{\alpha} B$ are empty if and only if $\sigma(A)$ is empty.

If $\sigma(A)$ is not empty, it holds

$$
\begin{aligned}
\sigma(A)+\sigma(B) & =\sigma\left(A \hat{\otimes}_{\alpha} I\right)+\sigma\left(I \hat{\otimes}_{\alpha} B\right) \\
& =\sigma(A \otimes I+I \otimes B)=\sigma\left(A \hat{\otimes}_{\alpha} I+I \hat{\otimes}_{\alpha} B\right) .
\end{aligned}
$$

Therefore, for any $\lambda \notin \sigma(A)+\sigma(B)$ (when $\sigma(A)$ is empty, $\lambda$ shall be an arbitrary complex number) and for any $f \in X \hat{\otimes}_{\alpha} Y$ there exists a unique $u \in D\left[A \hat{\otimes}_{\alpha} I\right] \cap D\left[I \hat{\otimes}_{\alpha} B\right]$ which satisfies

$$
\left(A \hat{\otimes}_{\alpha} I+I \hat{\otimes}_{\alpha} B-\lambda I \hat{\otimes}_{\alpha} I\right) u=f .
$$

Moreover the following inequality holds:

$$
\|u\|_{\alpha}+\left\|A \hat{\otimes}_{\alpha} I u\right\|_{\alpha}+\left\|I \hat{\otimes}_{\alpha} B u\right\|_{\alpha} \leq C\|f\|_{\alpha},
$$

with a constant $C$ independent of $u$ and $f$.

Proof. First note the condition of Theorem 3.1 is satisfied. Since $\sigma(A)+\sigma(B)$ is closed and does not cover the whole complex plane, it follows by Theorem 2.3 that $(A \otimes I+I \otimes B)^{\sim}=\left(A \hat{\otimes}_{\alpha} I+I \hat{\otimes}_{\alpha} B\right)^{\sim}$. So, to 
prove Theorem 3.2, it suffices to establish the inequality

$$
\left\|A \hat{\otimes}_{\alpha} I u\right\|_{\alpha} \leq C\left[\left\|\left(A \hat{\otimes}_{\alpha} I+I \hat{\otimes}_{\alpha} B\right) u\right\|+\|u\|_{\alpha}\right]
$$

for $u \in D[A \otimes I+I \otimes B] \equiv D[A] \otimes D[B]$.

Clearly, $-1 \notin \sigma(A)+\sigma(B)$. Then in virtue of Theorem 3.1, $(A \otimes I+$ $I \otimes B)^{\sim}+I \hat{\otimes}_{\alpha} I$ has an everywhere defined continuous inverse.

Since $A(A+(1+\eta) I)^{-1}=I-(1+\eta)(A+(1+\eta) I)^{-1}$ is uniformly bounded on $\sigma(B)$, the same argument as in the proof of Proposition 2.1 shows that the integral

$$
\begin{aligned}
\int_{\sigma(B)}[A(A & \left.+(1+\eta) I)^{-1} \otimes E(d \eta)\right] f \\
= & \int_{\sigma(B)}(A \otimes I)\left[(A+(1+\eta) I)^{-1} \otimes E(d \eta)\right] f
\end{aligned}
$$

defines a continuous linear operator of $X \otimes Y \subset X \hat{\otimes}_{\alpha} Y$ into $X \hat{\otimes}_{\alpha} Y$. It follows by the definition of the integral and by the closedness of $A \hat{\otimes}_{\alpha} I$ that $f \in X \otimes Y$ implies

$$
\left[(A \otimes I+I \otimes B)^{\sim}+I \hat{\otimes}_{\alpha} I\right]^{-1} f \in D\left[A \hat{\otimes}_{\alpha} I\right]
$$

and the integral (3.2) equals

$$
\left(A \hat{\otimes}_{\alpha} I\right)\left[(A \otimes I+I \otimes B)^{\sim}+I \hat{\otimes}_{\alpha} I\right]^{-1} f .
$$

Then we see that, for all $f \in X \hat{\otimes}_{\alpha} Y$, (3.3) holds valid and

$$
\left\|\left(A \hat{\otimes}_{\alpha} I\right)\left[(A \otimes I+I \otimes B)^{\sim}+I \hat{\otimes}_{\alpha} I\right]^{-1} f\right\|_{\alpha} \leq C\|f\|_{\alpha},
$$

whence follows immediately the inequality (3.1). Q.E.D.

Remark. In case $\left(\alpha_{2}\right)$, since an $\mathscr{L}_{2}$ space is considered as a Hilbert space and a normal operator is a scalar type spectral operator, it is possible to state the same assertion as in Theorem 3.2 for $B$ being a normal operator in $Y$. It should be emphasized that $A$ need not be $m$-accretive, in contrast to Theorem 4.6 (1) in [9]; this fact suggests that Theorem 3.2 is of wider application.

\section{Applications}

Theorem 3.2 is well applied to the first boundary value problem of a class of quasi-elliptic differential equations which includes especially the Laplace and heat equations (cf. [16], [9]). 
However, our theory is true in a far wider application; it includes the hyperbolic differential equations.

For an application of Theorem 3.1, we consider the initial value problem of the wave equation

$$
\left[\partial^{2} / \partial t^{2}-\partial^{2} / \partial x^{2}-\lambda\right] u(t, x)=f(t, x)
$$

in the strip $S=\left\{(t, x) \in R^{2} ; 0<t<T\right\}=I \times R, I=(0, T)$, with the initial condition

$$
u(0, x)=u_{t}(0, x)=0 .
$$

Then for any $\lambda \in C$ and for any $f \in L_{2}(S)=L_{2}(I) \hat{\otimes}_{\alpha_{0}} L_{2}(R)$ there exists a unique solution $u$ in $L_{2}(S)$ of the initial value problem (4.1) and (4.2); moreover we have $\|u\| \leq C\|f\|$, with a constant $C$.

To show this, let $A=d^{2} / d t^{2}$ in $L_{2}(I)$ with domain

$$
D[A]=\left\{\varphi \in L_{2}(I) ; d^{2} / d t^{2} \varphi \in L_{2}(I), \varphi(0)=\varphi^{\prime}(0)=0\right\}
$$

and let $B=-d^{2} / d x^{2}$ in $L_{2}(R)$ with domain

$$
D[B]=\left\{\psi \in L_{2}(\boldsymbol{R}) ;-d^{2} / d x^{2} \psi \in L_{2}(\boldsymbol{R})\right\} .
$$

Then $B$ is a selfadjoint operator in $L_{2}(R)$ with the spectrum $\sigma(B)$ being the nonnegative real line. $A$ is a densely defined closed linear operator in $L_{2}(I)$ with empty spectrum $\sigma(A)$. For $R>0$, let $U=\{\zeta ;|\operatorname{Im} \zeta|>R$ or $\operatorname{Rom} \zeta>R$.

Then we can show easily that $U+\sigma(B)=U \subset \complement K(0 ; R)$ and that $\|R(\zeta ; A)\| \leq C_{R}$ on $\complement U$, with a constant $C_{R}$ depending only on $R$. We denote by $L$ the operator $A \otimes I+I \otimes B$ with domain $D[A] \otimes D[B]$. Applying Theorem 3.1 yields the emptiness of the spectrum of the closure of $L=\partial^{2} / \partial t^{2}-\partial^{2} / \partial x^{2}$, whence follows the desired assertion. Here, we see that the solution $u$ lies in the domain of the closure of $L$.

\section{REFERENCES}

[1] Ju. M. Berezanskiĩ: Expansions in Eigenfunctions of Selfadjoint Operators, "Naukova Dumka", Kiev, 1965 (Russian) ; English transl., Transl. Math. Monographs vol. 17, Amer. Math. Soc., Providence, R.I., 1968.

[2] N. Dunford and J. T. Schwartz: Linear Operators, Part III. Spectral Operators, New York: Wiley-Interscience, 1971.

[ 3 ] B. Friedman: An abstract formulation of the method of separation of variables, Proc. Conf. Diff. Eqs., Univ. of Maryland, 1956, 209-226.

[ 4 ] A. Grothendieck: Produits tensoriels topologiques et espaces nucléaires, Memoirs Amer. Math. Soc., 16, 1955. 
[5] —_: Resumé de la théorie métrique des produits tensoriels topologiques, Bol. Soc. Mat. São Paulo, 8 (1956), 1-79.

[6 ] J. R. Holub: A note on P-spaces, Math. Ann., 193 (1971), 1-6.

[7] T. Ichinose: On the spectra of tensor products of linear operators in Banach spaces, J. Reine Angew. Math., 244 (1970), 119-153.

[8] - : Operational calculus for tensor products of linear operators in Banach spaces, to appear.

[ 9 ] - : Operators on tensor products of Banach spaces, Trans. Amer. Math. Soc., 170 (1972), 197-219.

[10] W. B. Johnson, H. P. Rosenthal and M. Zippin: On bases, finite dimensional decompositions and weaker structures in Banach spaces, Israel J. Math., 9 (1971), 488-506.

[11] G. Köthe: Topologische Lineare Räume. I, Berlin-Heidelberg-New York: Springer, 1966.

[12] — : General linear transformations of locally convex spaces, Math. Ann., 159 (1965), 309-328.

[13] J. Lindenstrauss and A. Płeczyński: Absolutely summing operators in $\mathscr{L}_{p}$-spaces and their applications, Studia Math., 29 (1968), 275-326.

[14] — and H. P. Rosenthal: The $\mathscr{L}_{p}$ spaces, Israel J. Math., 7 (1969), 325-349.

[15] L. Maurin and K. Maurin: Spektraltheorie separierbarer Operatoren, Studia Math., 23 (1963), 1-29.

[16] V. P. Mihaillov: On the first boundary problem for a class of hypoelliptic equations, Mat. Sb. 63 (105) (1964), 238-264 (Russian).

[17] M. Reed and B. Simon: A spectral mapping theorem for tensor products of unbounded operators, Bull. Amer. Math. Soc. 78 (1972), 730-733. Tensor products of closed operators on Banach spaces, to appear.

[18] R. Schatten: A theory of Cross-Spaces, Princeton Univ. Press, 1950.

Department of Mathematics

Hokkaido University 